\title{
Diferentes estrategias de tratamiento del Helicobacter pylori lograron similar eficacia de erradicación y riesgo de recurrencia
}

Different treatment strategies for Helicobacter pylori achieved similar eradication efficacy and recurrence risk rates

Douglas RM. JAMA 2013; 309(6):578-586.

\section{Objetivo}

Estimar el riesgo de recurrencia de infección por Helicobacter pylori y evaluar los factores asociados con la erradicación exitosa 12 meses después del tratamiento.

\section{Diseño, lugar y pacientes}

Estudio de cohortes, realizado en seis ciudades de América Latina (Chile, Nicaragua, Colombia, Costa Rica, Honduras y México), entre Septiembre de 2009 y Julio 2011. Se incluyeron individuos de entre 21 y 65 años, con infección confirmada para $\mathrm{H}$. pylori mediante el test de urea espirada (TUE), que no hubieran recibido tratamiento específico previo, ni sean portadores de patologías serias.

\section{Intervención}

Los pacientes fueron aleatorizado en tres grupos diferentes. El grupo Triple Esquema recibía durante 14 días 30 mg de lanzoprazol, $1 \mathrm{gr}$. de amoxicilina, y $500 \mathrm{mg}$ de claritromicina. El grupo Secuencial recibía durante cinco días $30 \mathrm{mg}$ de lanzoprazol más $1 \mathrm{gr}$. de amoxicilina, seguidos durante cinco días más de $30 \mathrm{mg}$ de lanzoprazol, $500 \mathrm{mg}$ de claritromicina y 500 $\mathrm{mg}$ de metronidazol $500 \mathrm{mg}$. El grupo Tratamiento Concomitante recibió durante cinco días $30 \mathrm{mg}$ de Lanzoprazol, $1 \mathrm{gr}$. de amoxicilina, $500 \mathrm{mg}$ de claritromicina y $500 \mathrm{mg}$ de Metronidazol. Todos los medicamentos eran genéricos y fueron administrados dos veces al día. Se contactaron a los pacientes a las ocho semanas de la aleatorización para evaluar efectos adversos, adherencia al tratamiento (definida como haber consumido más del $80 \%$ de los fármacos) y realizar un nuevo TUE. Pasados 12 meses de la fecha de inicio del estudio, los pacientes fueron nuevamente contactados para realizar un segundo TUE. Se definió como infección recurrente a aquellos pacientes con TUE negativo a las ocho semanas de haber realizado el tratamiento y un resultado positivo en el control de los 12 meses.

\section{Resultados principales}

En el control de los 12 meses, se obtuvo un riesgo de infección recurrente del 11,5\% (IC95\% 9,6\% a 13,5\%). Los factores asociados al riesgo de recurrencia fueron demográficos: cantidad de niños en edad escolar conviviendo (OR 1,17; IC95\% 1,01 a 1,35 ) y falta de adherencia al tratamiento (OR 2,94; IC95\% 1,31 a 6,13$)$, pero no hubo diferencia significativa según el esquema de tratamiento realizado. En cuanto a la efectividad del tratamiento según el régimen instaurado, los resultados fueron los siguientes: $80,4 \%$ en el grupo que recibió el Triple Esquema (IC95\% 76,4 a 83,9 ), $79,8 \%$ para el grupo que recibió la Terapia Secuencial (IC95\% 75,8 a 83,5), y $77,8 \%$ en el grupo que realizó la Terapia Concomitante (IC95\% 73,6 a 81,6).

\section{Conclusión}

El riesgo de infección recurrente luego del tratamiento para $\mathrm{H}$. pylori fue del $11,5 \%$, con variabilidad poco significativa entre los diferentes esquemas disponibles. Los factores asociados al éxito del tratamiento fueron sexo masculino, adherencia al tratamiento inicial, edad avanzada y sitio geográfico.

\section{Comentario}

El Colegio Americano de Gastroenterología, en su última guía del año 2007 recomienda buscar y tratar la infección por $\mathrm{H}$. pylori en los siguientes casos: enfermedad ulcero-péptica activa (ya sean úlceras gástricas o duodenales), antecedentes de enfermedad ulcero-péptica o linfoma gástrico tipo MALT y pacientes con carcinoma gástrico tratados quirúrgicamente.

La estrategia de buscar (con métodos no invasivos) y tratar al $\mathrm{H}$. pylori en pacientes menores de 55 años con síntomas de dispepsia, que no han sido estudiados, y que no presenten ningún signo o síntomas de alarma (como sangrado digestivo, anemia, saciedad precoz, pérdida de peso inexplicable, disfagia progresiva, vómitos, odinofagia recurrente y antecedentes personales o familiares de carcinoma gástrico) es también una opción válida. Sin embargo, esta recomendación esta efectuada por sociedades en las cuales la prevalencia de infección en su población es mucho más baja que en nuestro país (cercana al $50 \%$ ). Por lo tanto, dado que el riesgo de reinfección es más elevado en estos casos, debemos tener precaución y adecuar esta indicación al medio en el cual estemos.
En aquellos pacientes con dispepsia no ulcerosa, reflujo gastroesofágico, consumo habitual de AINEs, anemia ferropénica o población de alto riesgo para cáncer gástrico, la indicación de rastrear y tratar sigue siendo controversial ${ }^{1}$

La infección por $\mathrm{H}$. Pylori es un factor fundamental en el desarrollo de varias patologías gastrointestinales como úlceras gastroduodenales (en el 1 a 10\% de personas infectadas), linfoma gástrico tipo MALT (en menos del 0,01\%) y carcinoma gástrico (entre el $0,1 \%$ y $3 \%$ de los infectados). No obstante, es importante destacar que la gran mayoría de personas infectadas permanecerán asintomáticas. Si bien en ciertas regiones, los programas masivos para erradicación del $\mathrm{H}$. pylori pueden ser costo-efectivos, creemos que no se puede dejar de ver el riesgo de generar resistencia antibiótica en dichas regiones. Las políticas de salud pública en estos países deberían también poner su foco de atención en mejorar las condiciones higiénicas y sanitarias de la población, factor claramente asociado con el contagio de esta infección.

Yamila Mahumud [ Servicio de Medicina Familiar y Comunitaria del Hospital Italiano de Buenos Aires. yamila.mahumud@hospitalitaliano.org.armailto:yamila.mahumud@ hospitalitaliano.org.ar. mailto:yamila.mahumud@ hospitalitaliano.org.ar ]

Mahumud Y. Diferentes estrategias de tratamiento del Helicobacter pylori lograron similar eficacia de erradicación y riesgo de recurrencia. Evid Act Práct Ambul. 2014, 17(2). Abr-Jun. 58. Comentado de: Mongan. D et al. Risk of recurrent Helicobacter Pylori infection 1 year after initial eradication therapy in 7 Latin America Communities. JAMA 2013;309(6):578-586. PMID 23403682.

\section{Referencias}

1. Chey WD. et al. American College of Gastroenterology Guideline on the Management of Helicobacter pylori Infection. Am J Gastroenterology 2007;102:1808-1825.

2. Pest PS; et al Seroprevalencia de la infección por Helicobacter pylori en la República Argentina: influencia de la edad, sexo, nivel socio-económico, área geográfica e infraestructura sanitaria. Acta gastroenterol. Latinoam 1999;29(5):297-305. 